\title{
Les pèlerins et les fossiles de Voltaire
}

\author{
Par Marguerite Carozzi
}

Dans le domaine scientifique, la réputation de Voltaire a beaucoup souffert de son attitude négative en face des progrès rapides de son vivant des sciences de la terre. Son explication de la présence des fossiles marins loin de la mer par des pèlerins transportant et abandonnant des coquilles est souvent donnée comme exemple de cette attitude. Cette hypothèse est pourtant loin d'être aussi chimérique que généralement acceptée et mérite un examen approfondi. Dans le cadre du Bicentenaire de la mort de Voltaire, je propose un pèlerinage intellectuel aux sources de cette hypothèse. Une comparaison des six variantes révèle une évolution curieuse dans la pensée de Voltaire, une évolution régressive qui frappe par des détours de plus en plus compliqués pour éluder des questions scientifiques auxquelles Voltaire ne veut pas ou ne peut pas répondre et une imagination fertile pour adapter son hypothèse des pèlerins aux nouvelles connaissances en géologie.

\section{Saggio intorno ai Canbiamenti avvenuti su'l Globo della Terra, $1746^{1}$}

Le texte concernant les pèlerins est le suivant:

Fù scoperta fra i monti di Hassia una pietra, che portava il sembiante d'un Rombo. Sene conchiuse subito, che il Mare coprisse anticamente i monti di Hassia; senza darsi briga di congetturare, che quel rombo portato per la mensa d'un Signore di quel paese, si corrupe, fù gettato via, e poi s'impietrì. Un Luccio petrificato fù trovato sulla cima delle Alpi; i fumi dunque in un tempo correvano sù i monti, ed in un' altro la Germania era il seno del Mare!

Après un paragraphe à propos d'une ancre trouvée dans les montagnes de la Suisse et un autre sur les Glossopetrae et les ammonites, Voltaire mentionne des coquilles trouvées en Italie et en France:

In Italia, ed in Francia si ritrovano molte chiocciole, che passano per essere formate nei lidi di Soria. Non voglio dubitare punto della loro origine; ma i Filosofi potevano ricordarsi di quegl'innumerabili pellegrini, che andavano in frotta a guerreggiare, o vaneggiare anticamente verso la Palestina, dove portarono $i$ loro danari, e donde riportarono conchigliette $^{2}$. Non sò se sia meglio credere, che il Mare di Soria coprisse per un gran tempo Parigi, e Milano. Non sarebbe forse stravagante la congettura, che queste conche siano fossili. Molti Filosofi lo pensarano così; ma in qualunque opione, o errore che possiamo dare, non pare, che da queste chiocciole si possa arguire essere stato tutto il Mondo intieramente rovesciato. 


\section{$A V V E N U T I$ \\ $S$ U'L G L O B O \\ DELLA TERRA.}

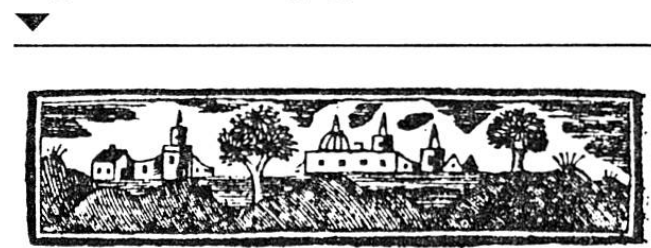

\section{$S A G G I O$}

I NTOR NO

\section{AI CANBIAMENTI}

\section{A V VENUTI}

$S$ U'L G L O B O

\section{DELLA TERRA.}

I fono errori popolari; vene fono Filofofici. Di quelto fer condo genere è forfe l'opiniome di molti Eruditi , che veggono, 0 credono di vedere fopra tutta la Terra manumenti d'una generale ruina, e diltruzzione.

Fù fcoperta fra imonti di Haffia una pietra, che portava il fembiante d'un Rambo. Sene conchiufe fubito, che il Mare copriffe anticamente i monti di Haflia; fenza darfi briga di congetturare, che quel rombo $A$ ij

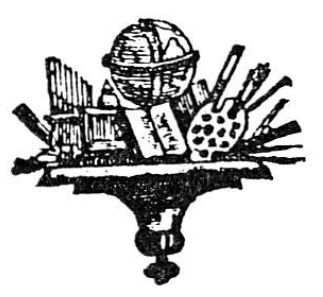

IN PARIGI,

Stampato da Pr a u l t fulla ripa di Gêvres.

M. D C C. X L VI.

\section{1}

vano anticamente sù l'erte cime dell' Alpi, che l'Oceano copriva la Germania ; e vedendo certe conchiglie affermerano, che il Mar di Soria è venuto a Francfort. Il gulto del meravigliofo produce i filtemi Itravaganti, ma la natura è altrettanto uniforme, femplice, e coltante, quanto le noAtre immaginazioni fono invaghite di prodi$\mathrm{gj}$, e di fegnalate revoluzioni.

VOITAIRE.

Aprile 1746.

Fig. 3. Dernière page 
A la fin du Saggio, Voltaire présente un résumé:

Sò bene che vi saranno sempre uomini, lo spirito de' quali sarà più percosso d'un luccio insassito sul monte Ceni, e d'un rombo petrificato in Hassia, che di tutti i ragionamenti della vera Filosofia. Si compiaceranno nell'immaginare, che i fiumi correvano anticamente sù l'erte cime dell'Alpi, che l'Oceano copriva la Germania; e vedendo certe conchiglie affermeranno, che il Mar di Soria è venuto a Francfort.

\section{Traduction du Saggio dans le Mercure de France, juillet 1746}

La traduction en français du Saggio fut publiée dans le Mercure de France en juillet 1746. Elle est précédée par l'introduction suivante: «Voici une traduction françoise dont l'original italien est d'un illustre Ecrivain François. M. de V. a composé ce morceau pour les Académies d'Italie, ausquelles il est agregé». Au sujet des pèlerins, voici le texte:

Quand on découvrit sur les montagnes de Hesse, une pierre qui avoit la figure d'un turbot, on en conclut qu'autrefois la mer avoit couvert ces montagnes. On ne daigna pas conjecturer que ce poisson fut porté là pour quelque repas \& qu'étant gâté on le jetta sur ces rochers, où depuis il s'étoit pétrifié. Un brochet pétrifié s'est trouvé sur la cime des Alpes. Il a donc été un tems où les fleuves ont coulé sur les montagnes, \& dans un autre tems l'Allemagne a été le sein de la mer.

Après la discussion de l'ancre, des Glossopetrae et des ammonites, Voltaire dit:

La France \& l'Italie sont pleines de petites coquilles qu'on prétend se former sur les côtes de Syrie. Je ne veux point revoquer en doute leur origine, mais les Philosophes ne pourroient-ils pas se rappeller cette multitude innombrable de Pelerins qui autrefois couroient en Palestine? On sçait qu'ils y porterent leur argent \& n'en rapporterent que des coquilles; vaut-il mieux croire que le terrain sur lequel Paris \& Milan sont bâtis ait servi pendant long-tems de lit à la mer de Syrie? Il ne seroit peut-être pas insensé d'avancer que ces coquilles sont fossiles. Plusieurs Philosophes l'ont cru, mais quelque système ou quelques rêveries que nous puissions adopter, il ne paroit pas possible de prouver par ces coquilles un renversement total du monde.

A la fin de la traduction, Voltaire dit:

Je sçais bien qu'il y aura toujours des hommes dont l'esprit sera plus frappé d'un brochet pétrifié sur le mont Cenis, \& d'un turbot trouvé dans le même état sur les montagnes de Hesse, que de tous les raisonnemens de la vraie Philosophie; ils se plairont à penser que les fleuves couloient autrefois sur la cime escarpée des Alpes, que l'Océan couvroit la Germanie, \& en voyant certaines coquilles, ils affirmeront que la mer de Syrie est venue à Francfort.

Dans le Saggio et dans la traduction de 1746, Voltaire mentionne deux genres de pétrifications: les poissons et les coquilles. Les poissons fossiles sont «un turbot» dans les montagnes de $\mathrm{Hesse}^{3}$ et «un brochet» sur la cime des Alpes, spécifiquement au Mont Cenis ${ }^{4}$. Des coquilles fossiles ont été découvertes en 


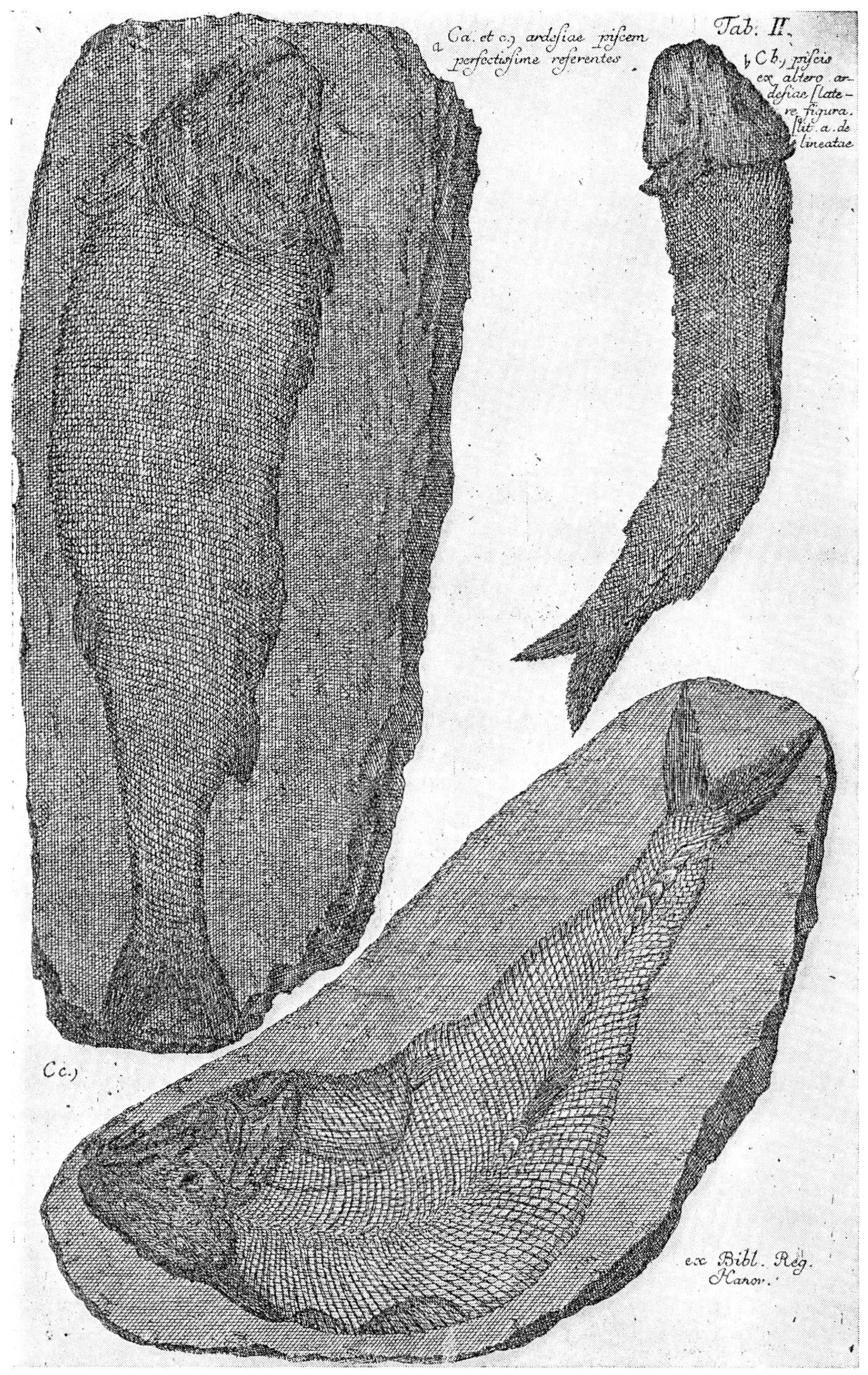

Fig. 4. Poisson fossile, Leibniz, Protogaea, Gottingen 1749, Table II 
Europe habité, en particulier à Paris ${ }^{5}$, à Milan ${ }^{6}$, et à Francfort ${ }^{7}$. La conclusion de certains philosophes, dit Voltaire, serait que la mer de Syrie ait envahi l'Italie, la France et l'Allemagne et que des fleuves et des mers aient coulé sur les Alpes. Voltaire suggère d'autres interprétations. Les poissons fossiles seraient des poissons gâtés et jetés par quelque voyageur, et pétrifiés par la suite ${ }^{8}$. Pour les coquilles fossiles, Voltaire présente trois interprétations: des coquilles d'espèces vivant actuellement sur les côtes de Syrie, ramassées et transportées par les pèlerins, ou des coquilles déposées en Europe quand la mer de Syrie couvrait ces régions. Quant à la troisième hypothèse, que ces coquilles sont «fossiles», il est impossible de savoir au juste ce que Voltaire voulait dire ${ }^{9}$.

L'analyse du Saggio et de sa traduction montre, d'une part, que Voltaire n'est pas prêt à accepter une interprétation des fossiles qui implique des bouleversements importants; d'autre part, que la présentation de son hypothèse est peu convaincante, c'est une idée qui lui a passé par la tête, une idée avancée d'un ton «un peu goguenard» comme il dira lui-même en 1767 (Moland XXVI: 408).

Ce ton peu scientifique n'a pas été apprécié par Buffon qui lisait le Saggio, dit-il, au moment même où il corrigeait son texte concernant les productions de la mer dans la Théorie de la Terre ${ }^{10}$. Dans cet ouvrage, il mentionne d'abord La Loubère qui «rapporte, dans son Voyage de Siam, que les singes, au cap de Bonne Espérance, s'amusent continuellement à transporter des coquilles du rivage de la mer au-dessus des montagnes ...»11 Ensuite Buffon attaque Voltaire:

En lisant une lettre italienne sur les changements arrivés au globe terrestre, imprimée à Paris cette année (1746), je m'attendais à y trouver ce fait rapporté par La Loubère; il s'accorde parfaitement avec les idées de l'auteur: les poissons pétrifiés ne sont, à son avis, que des poissons rares, rejetés de la table des Romains parce qu'ils n'étaient pas frais; à l'égard des coquilles, ce sont, dit-il, des pèlerins de Syrie qui ont rapporté, dans le temps des croisades, celles des mers du Levant qu'on trouve actuellement pétrifiées en France, en Italie et dans les autres Etats de la chrétienté. Pourquoi n'a-t-il pas ajouté que ce sont les singes qui ont transporté les coquilles au sommet des hautes montagnes et dans tous les lieux où les hommes ne peuvent habiter? cela n'eût rien gâté et eût rendu son explication encore plus vraisemblable. Comment se peut-il que des personnes éclairées et qui se piquent même de philosophie, aient encore des idées fausses sur ce sujet?

Tandis que Buffon semble bien comprendre le côté scientifique du Saggio où Voltaire distingue les poissons fossiles trouvés dans les montagnes des coquilles trouvées auprès des habitations humaines, il ne veut pas saisir le côté badin dans cet essai pseudo-scientifique de Voltaire. Buffon paraît prendre au sérieux la théorie de Voltaire (ou de La Loubère selon Buffon) du transport de coquilles 
par les pèlerins, d'où la nature violente de son attaque. Vu la grande popularité de l'œuvre de Buffon, il se pourrait bien que l'interprétation concernant les pèlerins ait été pris beaucoup plus au sérieux que ne l'entendait Voltaire luimême dans le Saggio. Au lieu de lire Voltaire, on a lu Buffon.

Dissertation envoyée par l'auteur, en italien, à l'Académie de Bologne et traduite par lui-même en français sur les Changements arrivés dans notre Globe et sur les Pétrifications qu'on prétend en être encore les Témoignages, 1748 (Moland XXIII: 219-230)

Le texte concernant les pèlerins est comme suit:

On a trouvé dans les montagnes de la Hesse une pierre qui paraissait porter l'empreinte d'un turbot, et sur les Alpes un brochet pétrifié: on en conclut que la mer et les rivières ont coulé tour à tour sur les montagnes. Il était plus naturel de soupçonner que ces poissons, apportés par un voyageur, s'étant gâtés, furent jetés, et se pétrifièrent dans la suite des temps: mais cette idée était trop simple et trop peu systématique (Moland XXIII: 221-222).

Voltaire mentionne ensuite l'ancre de vaisseau, les Glossopetrae et les ammonites comme en 1746 et continue ainsi:

On a vu aussi dans des provinces d'Italie, de France, etc. de petits coquillages qu'on assure être originaires de la mer de Syrie. Je ne veux pas contester leur origine; mais ne pourrait-on pas se souvenir que cette foule innombrable de pèlerins et de croisés, qui porta son argent dans la Terre Sainte, en rapporta des coquilles? Et aimera-t-on mieux croire que la mer de Joppe et de Sidon est venue couvrir la Bourgogne et le Milanais? On pourrait encore se dispenser de croire l'une et l'autre de ces hypothèses, et penser, avec beaucoup de physiciens, que ces coquilles, qu'on croit venues de si loin, sont des fossiles que produit notre terre. On pourrait encore, avec bien plus de vraisemblance, conjecturer qu'il y a eu autrefois des lacs dans les endroits où l'on voit aujourd'hui des coquilles; mais quelque opinion ou quelque erreur qu'on embrasse, ces coquilles prouvent-elles que tout l'univers a été bouleversé de fond en comble? (Moland XXIII: 222-223).

Vers la fin de la Dissertation, Voltaire ajoute:

Je sais bien qu'il se trouvera toujours des gens sur l'esprit desquels un brochet pétrifié sur le mont Cenis, et un turbot trouvé dans le pays de Hesse, auront plus de pouvoir que tous les raisonnements de la saine physique; ils se plairont toujours à imaginer que la cime des montagnes a été autrefois le lit d'une rivière ou de l'océan; quoique la chose paraisse incompatible; et d'autres penseront, en voyant de prétendues coquilles de Syrie en Allemagne, que la mer de Syrie est venue à Francfort (XXIII: 229).

En 1748 Voltaire sépare encore les deux genres de pétrifications - poissons et coquilles - mais l'hypothèse des poissons gâtés, jetés et pétrifiés est reléguée dans le passé («il était plus naturel»), apparemment rejetée par les physiciens parce 
qu'elle était trop simple et ne faisait partie d'aucun système qui aurait adopté un bouleversement majeur.

Les deux hypothèses de 1746 concernant les coquilles de Syrie sont répétées, à savoir celle d'un transport des coquilles par les pèlerins et celle d'une invasion de l'Europe par la mer de Syrie. Comme l'hypothèse de la mer de Syrie implique un bouleversement majeur, Voltaire présente une troisième interprétation, ainsi qu'en 1746, précisant toutefois que «ces coquilles, qu'on croit venues de si loin, sont des fossiles que produit notre terre.» Mais il n'est toujours pas certain si Voltaire pense à des organismes marins ayant vécu «dans notre terre» - dans un golfe ou une ancienne mer qui aurait avancée à l'intérieur de la terre et laissée des coquilles - ou s'il croit à un genre de génération spontanée ou un jeu de la nature. En opposant les coquilles «venues de loin» à des «fossiles que produit notre terre», il semble néanmoins plus probable qu'il a pensé à un golfe. Mais même un golfe représenterait encore le résultat d'un certain bouleversement et Voltaire introduit encore une quatrième hypothèse, celle des lacs couvrant les terrains fossilifères ${ }^{12}$. En 1748, Voltaire semble donner la préférence à cette dernière hypothèse.

\section{La Défense de mon Oncle, 1767 (Moland XXVI: 367-433)}

Dans le chapitre XIX, Des Montagnes et des coquilles, Voltaire attaque Buffon. Il dit au sujet des pèlerins:

Quand je lus, il y a quarante ans ${ }^{13}$ qu'on avait trouvé dans les Alpes des coquilles de Syrie, je dis, je l'avoue d'un ton un peu goguenard que ces coquilles avaient été apparemment apportés par des pèlerins qui revenaient de Jérusalem. M. de Buffon m'en reprit très-vertement dans sa Théorie de la Terre, page 281. Je n'ai pas voulu me brouiller avec lui pour des coquilles; mais je suis demeuré dans mon opinion, parce que l'impossibilité que la mer ait formé les montagnes m'est démontrée.

Il est dommage que Voltaire n'explique pas comment. Quoi qu'il en soit ce texte représente une satire où Voltaire fait des jeux de mots: il ne veut pas se brouiller avec Buffon pour des coquilles. En badinant, les poissons fossiles des Alpes sont devenus de simples coquilles; en fait, les poissons pétrifiés trouvés dans les Alpes ne seront dorénavant plus mentionnés.

Voltaire a minimisé la présence des poissons fossiles dans les montagnes dès 1746: c'étaient «un» brochet et «un» turbot. Pour expliquer les empreintes de poissons qui ressemblaient à ceux vivant actuellement dans la mer, Voltaire ne pouvait utiliser son interprétation d'un lac, ni d'un golfe, ni d'un pèlerin transportant des poissons pourris de la Terre Sainte - le Mont Cenis en particulier était haut placé et trop loin de la mer - et comme Voltaire voyait d'un mauvais œil 
la mer de Syrie causer des bouleversements importants, il a préféré, au début, l'hypothèse des poissons gâtés, jetés et pétrifiés. En 1767, Voltaire abandonne entièrement les faits d'observation de poissons fossiles en faveur des coquilles transportés par les pèlerins au sommet des montagnes.

Des Singularités de la Nature, chapitre XII - Des coquilles et des systèmes bâtis sur des coquilles, 1768 (Moland XXVII: 144-146)

...C'est une vérité reconnue, personne ne conteste les changements arrivés sur la surface du globe dans une longue suite de siècles. Il se peut physiquement, et sans oser contredire nos livres sacrés, qu'un tremblement de terre ait fait disparaître l'île Atlantide neuf mille ans avant Platon, comme il le rapporte, quoique ses Mémoires ne soient pas sûrs. Mais tout cela ne prouve pas que la mer ait produit le mont Caucase, les Pyrénées, et les Alpes.

On prétend qu'il y a des fragments de coquilles à Montmartre et à Courtagnon auprès de Reims. On en rencontre presque partout, mais non pas sur la cime des montagnes, comme le suppose le système de Maillet ${ }^{14}$. Il n'y a pas une seule sur la chaîne des hautes montagnes, depuis la Sierra-Morena jusqu'à la dernière cime de l'Apennin. J'en ai fait chercher sur le mont Saint-Gothard, sur le Saint-Bernard, dans les montagnes de la Tarentaise: on n'en a pas découvert.

Un seul physicien m'a écrit qu'il a trouvé une écaille d'huître pétrifiée vers le mont Cenis. Je dois le croire, et je suis très-étonné qu'on n'y en ait pas vu des centaines. Les lacs voisins nourrissent de grosses moules dont l'écail ressemble parfaitement aux huîtres; on les appelle même petites huîtres dans plus d'un canton. Est-ce d'ailleurs une idée tout à fait romanesque de faire reflexion sur la foule innombrable de pèlerins qui partaient à pied de Saint-Jacques en Galice, et de toutes les provinces, pour aller à Rome par le mont Cenis chargés de coquilles à leurs bonnets? Il en venait de Syrie, d'Egypte, de Grèce, comme de Pologne et d'Autriche. Le nombre de romipètes a été mille fois plus considérable que celui des hagi qui ont visité la Mecque et Médine, parce que les chemins de Rome sont plus faciles, et qu'on n'était pas forcé d'aller par caravanes. En un mot une huître près du mont Cenis ne prouve pas que l'océan Indien ait enveloppé toutes les terres de notre hémisphère.

On rencontre quelquefois en fouillant la terre des pétrifications étrangères, comme on rencontre dans l'Autriche des médailles frappées à Rome. Mais, pour une pétrification étrangère, il y en a mille de nos climats (Moland XXVII: 146).

Moland donne une version qui s'ajoutait, à l'origine, avant la phrase «On rencontre quelquefois...» (Moland XXVII: 146):

La chaîne des montagnes du continent américain n'est pas plus chargée d'huîtres que la nôtre, et la réponse qu'on en trouvera un jour n'est pas une réponse bien satisfaisante.

Mais il y a des fragments de coquillages à Montmartre, et à Courtagnon auprès de Reims.

Il y en a partout excepté sur les montagnes qui devraient en être remplies dans le système de Maillet. Oui sans doute, on l'a dit et il faut le redire, on rencontre quelque fois, en fouillant la terre; des pétrifications étrangères, comme on rencontre; etc. 
Cette version est plus affirmative («oui sans doute») et montre que c'était un dialogue à l'origine. Elle était aussi plus logique car les lignes à propos des pétrifications étrangères, "on rencontre...», devraient se trouver avec les nouvelles découvertes de Montmartre ${ }^{15}$ et de Courtagnon ${ }^{16}$ et non pas à la fin du paragraphe.

Une analyse de tous les chapitres mentionnant les fossiles dans Des Singularités de la Nature (Moland XXVII: 135-171) montre que Voltaire essaie de minimiser la présence de tous les fossiles: le falun de Touraine n'est pas une production marine mais simplement une «espèce de terre» sans coquilles visibles (Moland XXVII: 152), d'autres coquilles auraient pu être enfantées par le sol de la terre comme dans le domaine de M. Le Royer de La Souvagère (Moland XXVII: 148-149). Toutefois, dans le chapitre XII Voltaire est forcé d'admettre quelques faits d'observation: Il admet qu'on trouve des coquilles «presque partout»; il admet qu'en fouillant on trouve quelquefois des "pétrifications étrangères»; il admet qu'on a trouvé «une écaille d'huître pétrifiée vers le Mont Cenis»; d'autre part il ne conteste pas «les changements arrivés sur la surface du globe dans une longue suite de siècles».

Une comparaison avec les variantes précédentes montre un changement important dans la phrase-clef: les pèlerins ne reviennent plus de la Terre Sainte mais vont de Saint-Jacques de Compostelle, et de toutes les provinces, à Rome, par le Mont Cenis. Pourquoi ce revirement? Voltaire s'est-il rendu compte qu'en général les pèlerins de Compostelle rapportaient des coquilles Saint-Jacques, ceux allant à Rome revenaient avec des médailles et des indulgences, et ceux de la Terre Sainte avec des palmes de Jéricho ${ }^{17}$ ?

Une autre raison de ce revirement est peut-être son acceptation de quelques bouleversements mineurs - tremblements de terre, changements des côtes par suite d'affaissements de la terre ou de la diminution de la mer - des changements qui auraient permis aux golfes de la mer d'envahir des terres et d'y laisser des coquilles. (Voltaire dit dans le chapitre XI: «Quoi! parce que des atterrissements auront reculé la mer de plusieurs lieues, et qu'elle aura inondé d'un autre côté quelques terrains bas, on nous persuadra qu'elle a inondé le continent pendant des milliers de siècles!») A cause de cette nouvelle position, Voltaire n'a plus besoin des pèlerins transportant des coquilles dans le Milanais, les terrains de Paris, de Francfort et de Courtagnon car ces coquilles seraient, en fait, des fossiles locaux correspondant à la faune marine d'anciens golfes qui auraient envahi les terrains bas de l'Europe comme dans sa troisième hypothèse de 1748. Pour les rares pétrifications étrangères (une sur mille) Voltaire semble se référer à la foule des «romipètes» qui partaient de l'Espagne et de «toutes les provinces»; 


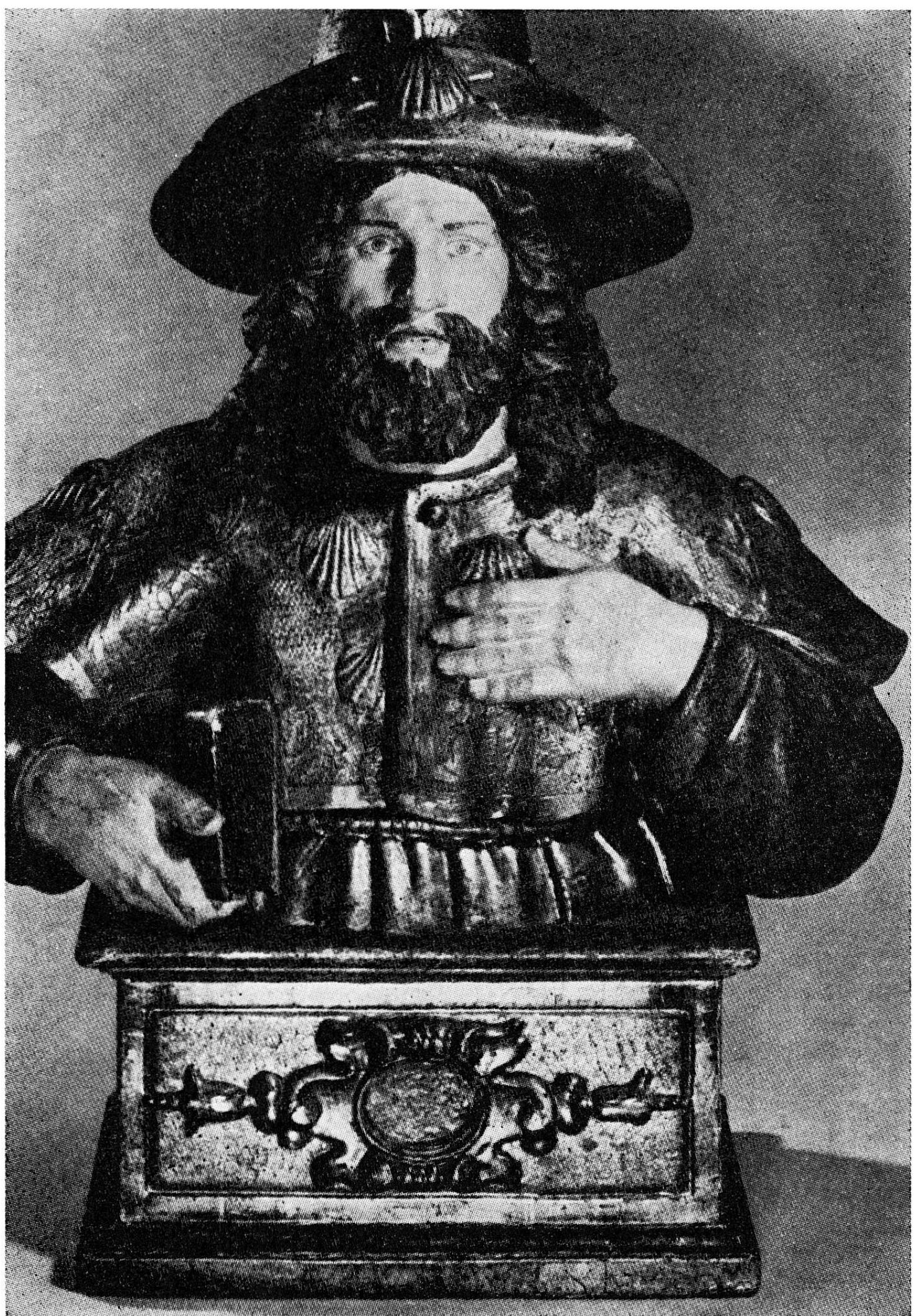

Fig. 5. Buste reliquaire de Saint-Jacques-de-Compostelle en bois doré, sur socle, Eglise de Cazères-sur-Garonne (H.-G.), reproduit d'après Pl. XII, Pèlerins et Pèlerinages dans les Pyrénées Françaises, Musée Pyrénéen, 1975 
mais Voltaire n'affirme rien, c'est au lecteur de faire cette association d'idées. Quant aux fossiles trouvés dans les montagnes, Voltaire affirme qu'il n'y en a pas - le Mont Cenis est une exception. Pour les fossiles qui s'y trouvent, il donne deux interprétations: les lacs et les pèlerins.

\section{L'Homme aux quarante Ecus, 1768 (Moland XXI: 305-368)}

Dans le chapitre VI, Voltaire s'entretient avec Telliamed qui demande:

- Mais, monsieur l'incrédule, que répondrez-vous aux huîtres pétrifiées qu'on a trouvées sur le sommet des Alpes?

- Je répondrai, monsieur le créateur, que je n'ai pas vu plus d'huîtres pétrifiées que d'ancres de vaisseau sur le haut du mont Cenis. Je répondrai ce qu'on a déjà dit ${ }^{18}$ qu'on a trouvé des écailles d'huîtres (qui se pétrifient aisément) à de très-grandes distances de la mer, comme on a déterré des médailles romaines à cent lieues de Rome, et j'aime mieux croire que des pèlerins de Saint-Jacques ont laissé quelques coquilles vers SaintMaurice ${ }^{19}$ que d'imaginer que la mer a formé le Saint-Bernard. Il y a des coquillages partout; mais est-il bien sûr qu'ils ne soient pas les dépouilles des testacées et crustacées de nos lacs et de nos rivières, aussi bien que des petits poissons marins?

- Monsieur l'incrédule, je vous tournerai en ridicule dans le monde que je me propose de créer.

- Monsieur le créateur, à vous permis; chacun est maître dans son monde; mais vous ne me ferez jamais croire que celui où nous sommes soit de verre, ni que quelques coquilles soient des démonstrations que la mer a produit les Alpes et le mont Taurus. Vous savez qu'il n'y a aucune coquille dans les montagnes d'Amérique. Il faut que ce ne soit pas vous qui ayez créé cet hémisphère, et que vous vous soyez contenté de former l'ancien monde: c'est bien assez.

- Monsieur, monsieur, si on n'a pas découvert de coquilles sur les montagnes d'Amérique, on en découvrira.

- Monsieur, c'est parler en créateur qui sait son secret, et qui est sûr de son fait. Je vous abandonne, si vous voulez, votre falun, pourvu que vous me laissiez mes montagnes. Je suis d'ailleurs le très-humble et très-obéissant serviteur de votre providence (Moland XXI: $332-333)$.

Dans cette dernière allusion aux pèlerins, Voltaire utilise le genre théâtral. En tirant galamment sa révérence, il déclare les fossiles être le problème des autres lui n'en a jamais vu. Ce sont des fables ou des imaginations de quelques faiseurs de système. Pourtant il n'oublie pas de répéter que les huîtres dans les hautes montagnes pourraient être lacustres ou apportées par les pèlerins. En ce qui concerne les coquilles dans les terrains habités, Voltaire redonne sa préférence à la quatrième hypothèse de 1748 , celle des lacs. Ne nous voilà pas plus renseignés sur ce que Voltaire considérait son hypothèse préférée. Je ne pense pas que ce soit celle des pèlerins. 
En comparant les textes qui accompagnent les six variantes de l'hypothèse des pèlerins, on constate que Voltaire tend à minimiser de plus en plus la présence des fossiles marins, soit dans les montagnes, soit dans les plaines. Il répète sans cesse que la mer n'a pas fait les montagnes. Pour avoir la paix, il admet dans son dernier acte l'origine marine du falun de Touraine pourvu qu'on lui laisse ses montagnes. Voltaire semble s'accrocher à une prétendue stabilité des montagnes autant que les naturalistes de son temps essayaient de démolir ce mythe. J'ai l'impression que Voltaire a gardé son hypothèse des pèlerins par simple obstination en face d'une science qui ne pouvait pas lui apporter des réponses à toutes ses questions, surtout celle de la formation des montagnes. Faute de mieux, il préférait croire à sa propre imagination, à ses propres rêves, car Voltaire n'est-il pas poète avant tout? Voici ce qu'il disait à propos des pèlerins:

Le Poitevin prend le chemin d'Ancône

Avec sa dame, un bourdon dans la main,

Portant tous deux chapeau de pèlerin,

Bien relevé de coquilles bénies... (Moland IX: 143).

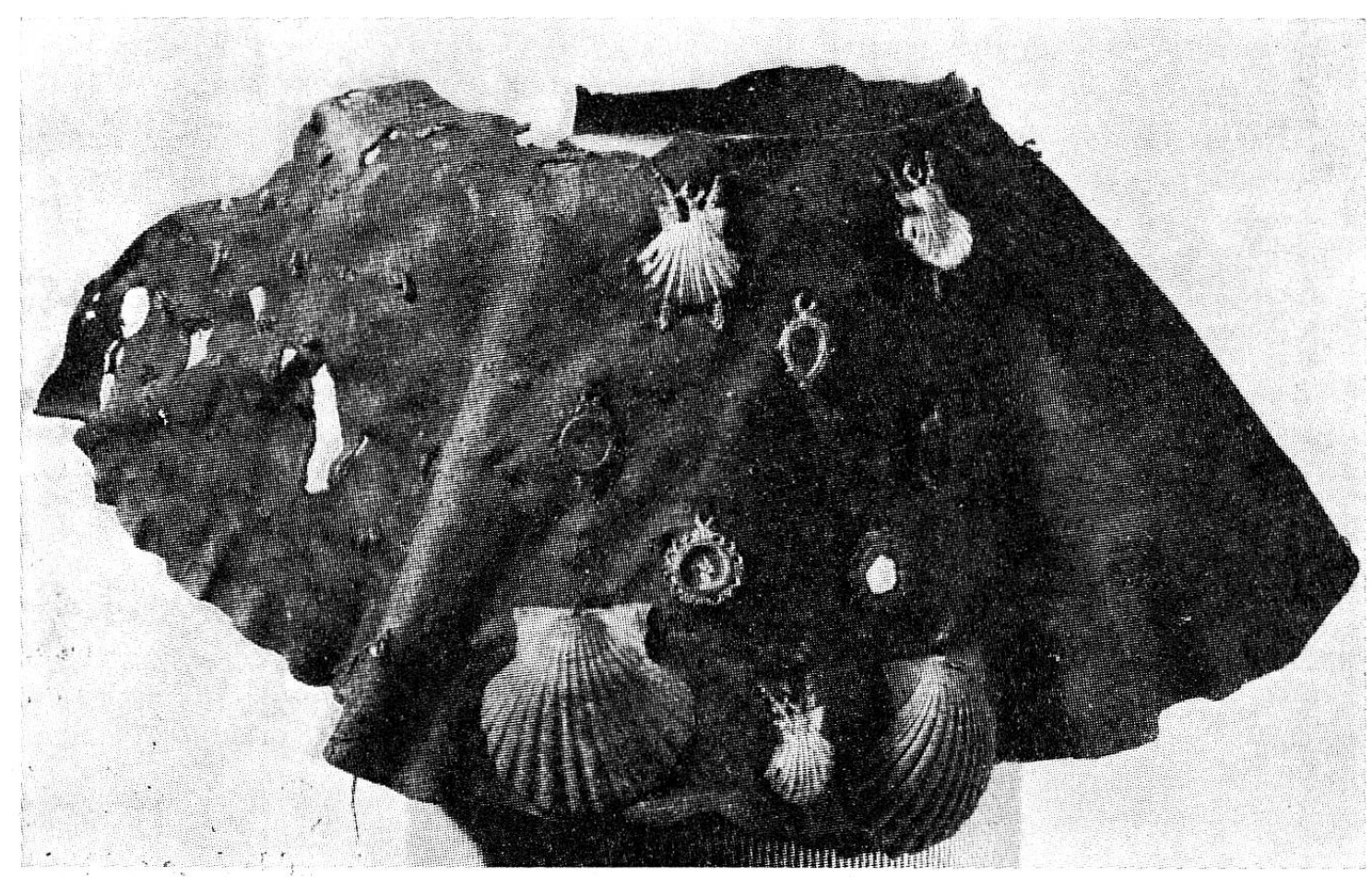

Fig. 6. Esclavine ou pèlerine de cuir, XVII ${ }^{\mathrm{e}}$ siècle, médailles en plomb, coquilles d'argent. Trouvée dans une tombe de l'ancienne cimetière de Lourdes. Reproduit d'après Pl. XI, Pèlerins et Pèlerinages dans les Pyrénées Françaises, Musée Pyrénéen, 1975 
J'aimerais remercier ici Monsieur Charles Wirz d'avoir bien voulu me procurer la photocopie du Saggio dont une édition originale se trouve au musée Voltaire. Mes remerciements vont également à Monsieur Edouard Lanterno et Monsieur Slatkine, père, pour leur assistance précieuse.

Les Euvres complètes de Voltaire, mentionnées dans le texte même, sont de l'édition Louis Moland, Paris 1877-1885, 52 vol.

${ }^{1}$ Les citations en italien et en français sont conformes aux textes originaux. Aucune faute d'orthographe ou grammaticale n'a été corrigée.

2 Afin de faciliter la comparaison des six variantes, la phrase-clef de chacune des variantes est mise en italique. Les autres phrases ou mots cités en italique sont conformes aux textes originaux.

${ }^{3}$ Il s'agit probablement d'un poisson fossile du fameux gisement nommé «Fischschiefer» de Sollingen (Rupelton ou «Septarienton», Oligocène). Cf. Oscar Speyer, Zeitschrift der Deutschen Geologischen Gesellschaft, vol. XII, Berlin 1860, p. 507; et Friedrich Schwarz in Kassel, Abhandlungen der Preußischen Geologischen Landesanstalt - «Geologie der Gegend zwischen Kassel und dem Reinhardswalde», Berlin 1930, Heft 132, p. 25-26.

Voltaire a peut-être lu la Protogaea de Leibniz dont un premier aperçu a été donné dans le Journal de Leipsick (Acta eruditorum) en janvier 1693 selon Bertrand de Saint-Germain, Protogée ou de la Formation et des Révolutions du Globe par Leibniz, Paris 1859, p. ii-iii.

Leibniz y décrit des poissons fossiles des carrières d'ardoises de Eisleben et d'Osterode (Osterode est à côté de Sollingen) comme suit (p. 47): «J'ai entre les mains des fragments reproduisant le mulet, la perche, l'able, gravés dans la pierre. Peu auparavant, on avait retiré de la carrière un énorme brochet, le corps fléchi, la bouche ouverte, comme s'il eut été surpris vivant et qu'il fut roidi contre la force pétrifiante. J'ai vu aussi des poissons de mer comme la raie, le hareng, la lamproie, et celle-ci souvent couchée sous le hareng.» Voltaire a peut-être même vu le brochet exposé à la bibliothèque de Hanovre (voir photo ci-dessus) pendant un de ses voyages à Berlin en 1740 et 1743.

${ }^{4}$ Reynold Barbier (Les Zones Ultradauphinoise et Subbriançonnaise entre l'Arc et l'Isère Mémoires pour servir à l'explication de la carte géologique détaillée de la France, Paris 1948) mentionne deux importants gisements fossilifères dans cette région: la faune rhétienne dans le Pas du Roc, en aval de Saint-Michel-de Maurienne, dans la vallée de l'Arc (p. 40-43), et la faune liasique de la "grosse pierre des Encombres», un bloc de presque quarante mètre de long, tombé d'une paroi de calcaires du Lias et se trouvant maintenant au fond du «talweg» (p. 52-56, 216). Barbier indique que les fossiles identifiables sont devenus introuvables après "près d'un siècle qu'on vient y récolter des fossiles", surtout sur la fameuse pierre des Encombres. Ce n'est toutefois que dans le Rhétien du Pas du Roc que l'Abbé Vallet a signalé en 1860 des couches à débris de poissons (Bulletin de la Société Géologique de France, tome 18, $2^{\text {e }}$ série, 1860-1861, p. 693-798-826). L'Abbé Antoine Stoppani a identifié ces fossiles parmi lesquels il y a deux dents de poissons (Paléontologie Lombarde, Milan, 1860-1865, p. 197-200, Pl. 34). Il semble donc qu'il n'y ait pas eu au Mont Cenis un gisement important de poissons fossiles entiers comme par exemple à Bolca, à Oeningen ou au canton de Glaris, mentionnés par L. Bourguet en 1742 (Traité de Pétrifications, Paris, p. 120-121). Par contre les fossiles étaient probable- 
ment abondants et bien visibles du temps de Voltaire, et il est probable qu'on ait pu y trouver un poisson fossile entier; mais je n'ai pas, jusqu'à présent, pu trouver la source précise.

${ }^{5}$ Il s'agit probablement de Issy-les-Moulineaux, à quelques kilomètres de Paris (Montien avec de nombreux fossiles de mollusques, d'échinodermes et de coraux) mentionné par Réaumur dans son article, "Sur les Coquilles Fossiles de quelques Cantons de Touraine, \& sur les utilités qu'on en tire» dans Histoire de l'Académie Royale des Sciences, Amsterdam $1720 ;$ publ. 1724, p. 538. De Maillet signale aussi un banc de coquillages à Issy dans Telliamed ou Entretien d'un Philosophe Indien avec un Missionnaire François sur la Diminution de la Mer, la Formation de la Terre, l'Origine de l'Homme, etc., Amsterdam 1748, et dans le manuscrit qui circulait pendant vingt ans avant la publication du livre. (En ce qui concerne la circulation du manuscrit voir Lamoignon de Malesherbes, Observations de Lamoignon-Malesherbes sur l'Histoire Naturelle Générale et Particulière de Buffon et Daubenton, Tome premier, Paris, An VI (1798), p. 222). Voltaire possédait dans sa bibliothèque de Ferney l'édition d'Amsterdam 1748 de Telliamed (George R.Havens and Norman L.Torrey, Voltaire's catalogue of his library at Ferney, SVEC, vol. IX, Genève 1959, p. 41) et le manuscrit Nouveau système du monde ou entretien de Teliamede (p. 33 et 184 selon le catalogue ci-dessus).

Voltaire mentionne le manuscrit et le livre de de Maillet dans le Catalogue de la plupart des Ecrivains Français qui ont paru dans le siècle de Louis XIV pour servir à l'histoire littéraire de ce temps (Moland XIV: 99-100), et il est certain que déjà dans le Saggio beaucoup d'allusions s'adressent à de Maillet. Telliamed a été traduit et édité par Albert V. Carozzi, Telliamed or Conversations Between an Indian Philosopher and a French Missionary on the Diminution of the Sea by Benoit de Maillet, University of Illinois Press, Urbana 1968, une traduction qui est basée sur le manuscrit que Voltaire a pu lire avant 1748. Dans cet ouvrage, de Maillet mentionne Issy à la page 100 .

${ }^{6}$ A Milan ou plutôt dans le Milanais, la région de Tortona (Stazzano, Sant'Agata dei fossili) est riche en marnes bleues (Tortonien) qui contiennent de nombreux fossiles. Cf. Maurice Gignoux, Géologie Stratigraphique, $2^{\mathrm{e}}$ éd., Paris 1936, p. 500 ; Congrès Géologique International - Commission de Stratigraphie: Lexique stratigraphique International, Volume I: Europe, Centre National de la Recherche Scientifique, Italie, Paris 1955, p. 129; Geologia dell'Italia, Ardito Desio, Torino 1973, p. 543.

${ }^{7}$ Il s'agit probablement du calcaire fossilifère (Miocène) du Bassin de Mainz à Sachsenhausen près de Francfort mentionné par J.A. de Luc, Geological Travels in Some Parts of France, Switzerland and Germany, F. C. and J.Rivington, London 1813, I, p. 244. Voir la traduction de Albert V. Carozzi de Telliamed, p. 119 et 292.

Voltaire a pu trouver cette indication dans le manuscrit de de Maillet (édité et traduit par A.V.Carozzi), p. 100.

${ }^{8}$ Cette théorie ressemble un peu à celle avancée par Bernard Palissy, en 1563, dans Recepte Veritable..., Euvres complètes, Paris 1961, p. 37: «Pres dudit fossé il y a eu d'autres fois quelque habitation, et ceux qui pour lors y habitoient, apres qu'ils avoient mangé le poisson qui estoit dedans la coquille, ils jettoient lesdites coquilles dedans la vallée, où estoit ledit fossé, et par succession de temps, les dites coquilles s'estoient dissoutes en la terre...» Voltaire parle d'empreintes de poissons entiers et son interprétation est légèrement différente; mais les deux auteurs partagent l'opinion que quel- 
ques personnes auraient pu jeter une partie de leur repas qui se serait fossilisée par la suite en dehors de la présence de la mer.

${ }^{9}$ Le mot «fossile » était utilisé jusqu’à la fin du dix-huitième siècle pour désigner toutes les substances qui se tiraient du sein de la terre. On distinguait deux genres: les fossiles propres (ou natifs) à la terre comme les pierres, les cristaux, les métaux, etc. et les fossiles étrangers à la terre comme les coquilles, les poissons, les plantes, etc. qu'on trouvait à l'intérieur de la terre où ils avaient été apportés accidentellement. Voir l'article «Fossile» dans Encyclopédie ou Dictionnaire Raisonné des Sciences, des Arts et des Métiers..., éd. Diderot, Paris MDCCLVII, Tome septième, p. 209.

${ }^{10}$ Georges-Louis Leclerc, comte de Buffon, Euvres complètes, tome 1, nouvelle édition, Poulain et Cie, Paris 1850-1860, p. 161.

${ }^{11}$ Simon de La Loubère, envoyé extraordinaire du roi de France au roi de Siam pendant les années 1687 et 1688, est l'auteur du livre Du royaume de Siam (2 vol., publié à Paris et Amsterdam en 1691, traduit et publié à Londres en 1693). La version anglaise a été rééditée avec une introduction par David K. Wyatt, Oxford University Press, 1969. Dans cette dernière édition, La Loubère dit dans la description du Cap de Bonne Espérance: "Some of our Crew went to the top of the Table Mountain, to seek some extraordinary Plants, but they found none. Nevertheless, upon strict Scrutiny, there is not any that has not something particular, which the Plants of these Countries have not. The shells there found are not Remains of the Deluge, as some have conjectur'd. The Birds, the Apes, and the Hotantots, do bring them, and leave them there" (Tome II, p. 183).

Voltaire mentionne La Loubère dans le Catalogue... (op. cit. Moland XIV : 85) en disant, «On a de lui des Mémoires de ce pays meilleurs que ses sonnets et ses odes». Voilà peut-être la source des pèlerins transportant des fossiles: les Hottentots sont devenus les pèlerins de Voltaire.

12 L'hypothèse des lacs est discutée par Palissy (op. cit. p. 277-280) et par Leibniz (op. cit. p. 48).

${ }^{13}$ Il s'agit probablement du manuscrit de Telliamed qui signale la présence de poissons fossiles trouvés loin de la mer qui ressemblent parfaitement à ceux pêchés sur les côtes de la Syrie (Cf. édition Albert V.Carozzi, p. 89). Toutefois de Maillet ne dit pas qu'on a trouvé des coquilles de Syrie dans les Alpes.

${ }^{14}$ Une lecture approfondie de Telliamed montre que dans les premières montagnes, après la diminution de la mer, il y a eu peu ou point de fossiles d'organismes "puisqu'il n'y avait alors aucuns terrains voisins de la superficie de la mer seule capable de leur fournir la nourriture nécessaire» (p. 76, édition Amsterdam 1748), et c'est seulement dans les montagnes "postérieures» que l'on les trouve en abondance (p. 78, op. cit.). Par contre, à deux reprises (p. 34 et 72) de Maillet se contredit. Ou Voltaire a lu Telliamed d'une façon superficielle, ou il s'adresse non pas à de Maillet mais à ceux qui ont adopté le «système de Maillet» sans faire aucune recherche sur les lieux.

${ }^{15}$ Les sables de Fontainebleau dans la Butte-Montmartre contiennent des marnes à Huîtres. Cf. Ch. Pomerol, Guides Géologiques Régionaux - Bassin de Paris, Paris 1974, p. 37.

${ }^{16}$ Cf. Buffon, op. cit. p.163; Bourguet, op. cit. Indice p. 31.

${ }^{17}$ Cf. Raymond Oursel, Les Pèlerins du Moyen Age, Les Hommes, Les Chemins, Les Sanctuaires, dans Résurrection du Passé, Payard 1963, p. 99. 
K. Baedeker, Palestine et Syrie - Manuel du Voyageur, $2^{\mathrm{e}}$ éd., Leipzig 1893, p. 167-172, 235-237.

Claude Pasteur, De Chartres à Jérusalem, La Palatine, Paris 1968, p. 135-180.

Convegni del Centro di Studi sulla spiritualità Medievale IV, Pellegrinaggi e Culto dei Santi in Europa fino alla Ia Crociata, Aprile 1961 in Todi presso l'Accademia Tuderina, 1963, p. 115-144.

Le Guide du Pèlerin de Saint-Jacques de Compostelle, Texte latin du XII siècle; édité et traduit par Jeanne Vieilliard, $2^{\mathrm{e}}$ éd., Macon MCML.

Romain Roussel, Les Pèlerinages, Edition «Que sais-je?», Presses Universitaires de France, Paris 1972, 172 p.

F. Raphael et al., Les Pèlerinages de l'antiquité biblique et classique à l'occident médiéval Etude d'Histoire des Religions No.1, Paris 1973, 173 p.

Pèlerins et Pèlerinages dans les Pyrénées Françaises, Musée Pyrénéen, Exposition JuinOctobre 1975, 88 p. avec illustrations.

Je n'ai trouvé aucune mention de pèlerins revenant de la Terre Sainte avec des coquillages comme témoignages de leur voyage alors que les coquilles Saint-Jacques, ramassées par les pèlerins au bord de l'océan, sont les insignes-témoins d'un rite que chaque pèlerin doit accomplir quand il va à Saint-Jacques de Compostelle. Toutefois, la tradition a pu se perdre et de nombreux pèlerins portaient probablement la coquille Saint-Jacques sans avoir été en Espagne. Nous avons rencontré, par exemple, en revenant de Genève cet été, de jeunes gens de Milwaukee avec des coquilles Saint-Jacques dorées comme pendentifs. Ils revenaient d'Israël où ils s'étaient baignés dans la mer à Césarée. L'un des voyageurs avait même ramassé un vrai pecten au bord de la mer et le portait suspendu avec la coquille dorée. Ils se souvenaient vaguement que la coquille représente un objettémoin du baptême chrétien mais ignoraient l'origine de cette tradition.

18 Apparemment dans Des Singularités de la Nature.

${ }^{19}$ Une localité nommé Bourg Saint-Maurice se trouve à côté du Saint-Bernard.

\section{Summary}

The author quotes and discusses the different passages where Voltaire tried to explain the presence of marine fossils far from the ocean. Voltaire did not believe that the sea had once covered the mountains. He thought that the fishes had been thrown away by travellers and that the shells had been brought back by pilgrims from the Holy Land or from Spain, so that they could be found in the soil like old Roman coins.

\section{Marguerite Carozzi}

709 West Delaware

Urbana, Illinois, 61801

USA 